\title{
Analysis of Self-standing Structure composed by Thick Resist Layer
}

\author{
Akihiro Takano and Akira Kawai* \\ Department of Electrical Engineering, Nagaoka University of Technology, \\ 1603-1 Kamitomioka, Nagaoka, Niigata 940-2188, Japan \\ (*Corresponding author, kawai@nagaokaut.ac.jp)
}

A self-standing resist (SSR) film in hexagonal lattice structure is fabricated and tested its destruction strength. The resist film formed on a glass substrate is removed from the glass substrate by wet etching. As a result, higher destruction strength of the SSR film with micro-hexagonal hole array can be obtained compared with that without holes.

Keywords: self-standing structure, hexagonal lattice structure, photoresist, destruction strength, MEMS

\section{Introduction}

MEMS (Micro Electro Mechanical systems) technology has been widely employed for fabricating of several micro devices such as inkjet printer head, acceleration sensor, biochip and so on.[1-4] Recently, polymer materials, such as photoresist resin, have been focused as permanent structural materials used for MEMS devices. It is required that the permanent structural materials are durable to employ to MEMS device component. In this regard, we have tried to fabricate a selfstanding structure of more strong and flexible. In this paper, it is demonstrated that the mechanical strength of self-standing resist (SSR) film is enhanced by forming hexagonal micro hole array. The destruction strength of the SSR film is analyzed by peel destruction test.

\section{Experiment}

2.1 Fabrication of SSR film

As a SSR film material, epoxy resin based negative photoresist in commercial available was used. The mechanical properties of the resist film was summarized in Table-1.[5] Figure 1 shows an artificial ultra violet (UV) exposure system. It was constructed with an UV source $(\lambda=365 \mathrm{~nm})$, a photomask holder, a X-Y stage, a microscope for adjusting a position of as-coated resist film. The

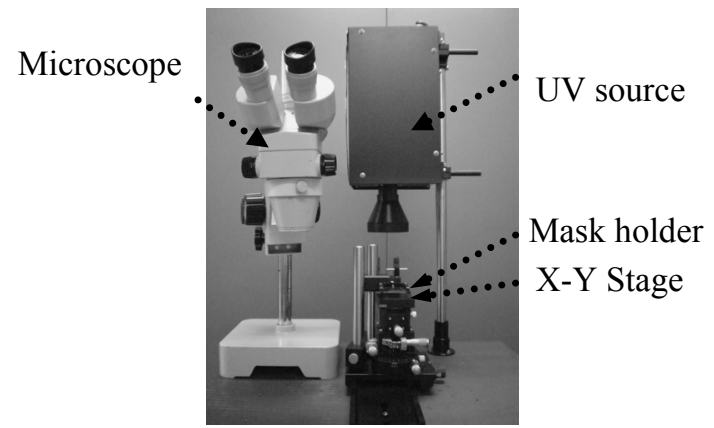

Fig.1 UV exposure system for SSR structure fabrication.

(a)

(b)

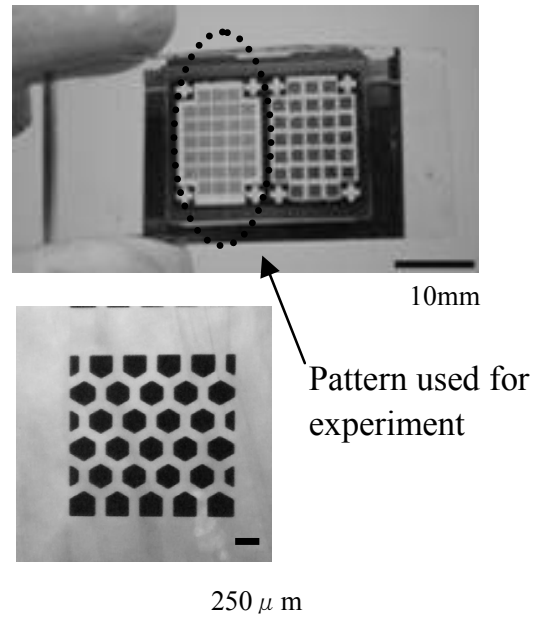

Fig.2 Photographs of glass dry plate photomask. (a) mask plate, (b) enlarged view of hexagonal pattern. 
Table1 Mechanical properties of epoxy resin based negative photoresist film. The test conditions are as follows: baking at $95^{\circ} \mathrm{C}$ for $15 \mathrm{~min}$, exposing to i-line, post exposure baking at $95^{\circ} \mathrm{C}$ for $6 \mathrm{~min}$ and hard baking at $150^{\circ} \mathrm{C}$ for $60 \mathrm{~min}$.

\begin{tabular}{|l|c|}
\hline Tensile strength $(\mathrm{MPa})$ & 55.0 \\
\hline Young's Modulus $(\mathrm{Mpa})$ & 2050 \\
\hline Elongation $(\%)$ & 4.0 \\
\hline Glass transition temperature $\left({ }^{\circ} \mathrm{C}\right)$ & 200 \\
\hline Thermal Stability $\left({ }^{\circ} \mathrm{C}\right)$ & 300 \\
\hline
\end{tabular}

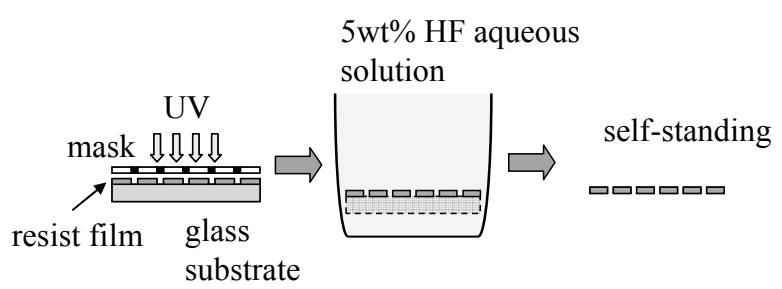

Fig.3 Fabrication process of SSR structure (cross sectional view).

photograph of photomask and its enlarged view were shown in Figure 2. A photomask and a resist coated substrate were placed at the X-Y stage. Then, they were aligned each other using the microscope. The fabrication process of the SSR structure is as follows. Figure 3 shows a schematic flowchart of the SSR fabrication process. The resist film was coated on a glass substrate in $100 \mu \mathrm{m}$ thickness by spin coat method. The resist film was heated on a hotplate at $60^{\circ} \mathrm{C}$ for $5 \mathrm{~min}$ and then baked at $95^{\circ} \mathrm{C}$ for $10 \mathrm{~min}$. The resist film was contacted with the photomask and was exposed to UV light for 8s. Post exposure bake was carried out on the hotplate at $60^{\circ} \mathrm{C}$ for $2 \mathrm{~min}$ and subsequently it was baked at $95^{\circ} \mathrm{C}$ for $6 \mathrm{~min}$. Finally, the resist film was dipped into $5 \mathrm{wt} \%$ hydrofluoric (HF) aqueous solution for $5 \mathrm{~h}$ in order to remove the glass substrate from the resist film. Then the resist film was rinsed in deionized water.

\subsection{Destruction strength analysis of SSR film}

Figure 4 shows the test system of destruction strength for the SSR film. The one side of the SSR film was fixed by a clip. Then, a weight was suspended from the another side of the SSR film. The weight gradually increased until the breaking point of the SSR film. The destruction strength $F$ of SSR film was defined as gravity at the breaking point as follows:

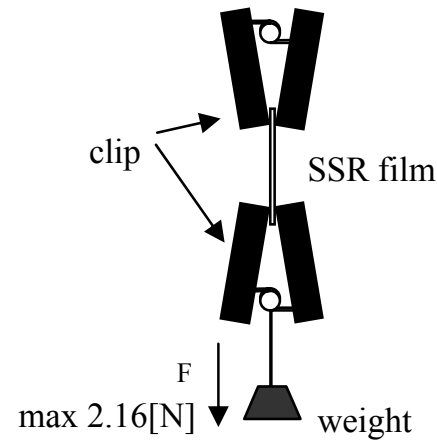

Fig.4 Experimental system of destruction strength analysis. (a)

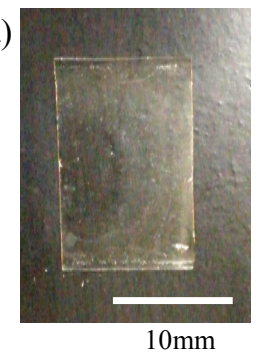

(b)

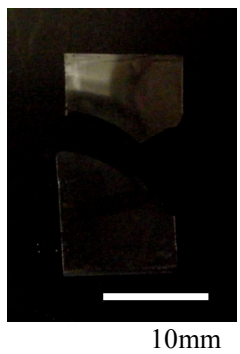

(c)

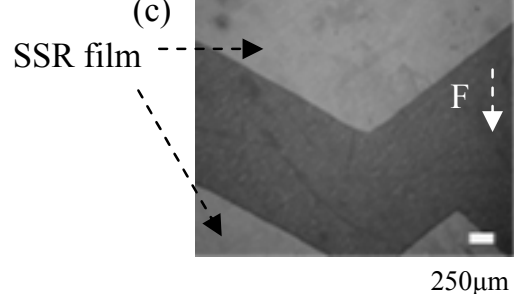

Fig.5 Photographs of SSR film without patterning. (a) before destruction, (b) after destruction, (c) enlarged view of SSR film after destruction. (a)

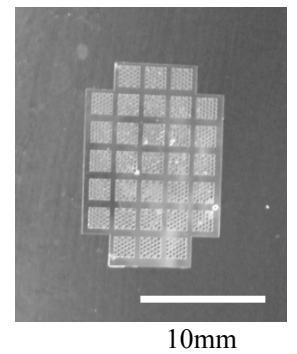

(c)

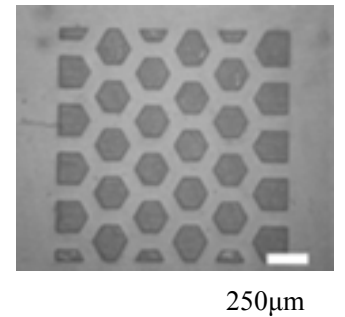

(b)

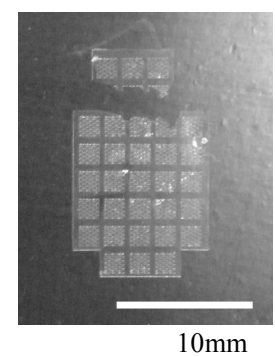

(d)

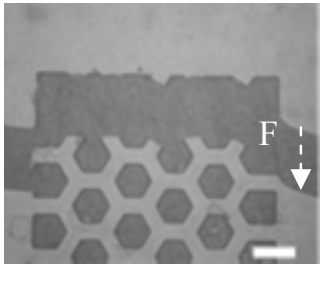

Fig.6 Photograph of patterned SSR film. (a) before destruction, (b) after destruction, (c) enlarged view of SSR film before destruction, (d) enlarged view of SSR film after destruction. 


$$
F=m g \quad[\mathrm{~N}],
$$

where symbols $m$ and $g$ are weight mass and gravitational acceleration, respectively. The peel strength $\sigma$ as the destruction strength per unit area was given by

$$
\sigma=F / S=m g / S[\mathrm{~Pa}],
$$

where a symbol $S$ is the cross sectional area of the SSR film.

\section{Results and Discussion}

The photograph of SSR film without hexagonal hole array is shown in Figure 5a. The size of SSR film is $10 \mathrm{~mm} \times 19 \mathrm{~mm}$. Figures $5 \mathrm{a}$ and $5 \mathrm{~b}$ show the SSR film before and after the destruction, respectively. An enlarged view of the cohesive failure area is shown in Figure $5 \mathrm{c}$. The peel strength of resist film is $0.59 \mathrm{MPa}$ and destruction strength is $0.59 \mathrm{~N}$ (cross sectional area $S=10^{-6} \mathrm{~m}^{2}$ ). Figure 6 shows the SSR film with micro hole patterning. Figures $6 \mathrm{a}$ and $6 \mathrm{~b}$ show the photographs of the resist film before and after the destruction, respectively. The enlarged photographs are also shown in Figures $6 \mathrm{c}$ and $6 \mathrm{~d}$. As shown in Figure $6 \mathrm{c}$, the hexagonal lattice structure in SSR film is clearly observed. In Figure $6 \mathrm{~d}$, the destruction mainly occurs at the end line of hexagonal lattice array. The peel strength of SSR film with hexagonal lattice is $2.16 \mathrm{MPa}$ and the destruction strength is $2.16 \mathrm{~N}$. These results are plotted in Figure 7 with destruction strength on the vertical axis and structure size on horizontally.

In general, tensile stress $\sigma$ is given by Hook's law:

$$
\sigma=E \varepsilon
$$

where $E$ and $\varepsilon$ are Young's modulus and strain, respectively. Young's modulus represents the stiffness of material. Higher Young's modulus value indicates higher resistant to tensile deformation. As shown in Table 2, epoxy resin indicates higher Young's modulus among polymer materials.[8] Therefore, it seems that the epoxy based resist is suitable to apply to MEMS fabrication as a permanent material.

The peel strength of the SSR film with hexagonal hole array is about four times as high as the SSR without patterning. For this reason, it can be considered that the hexagonal lattice patterns formed in SSR film acts to enhance the durability

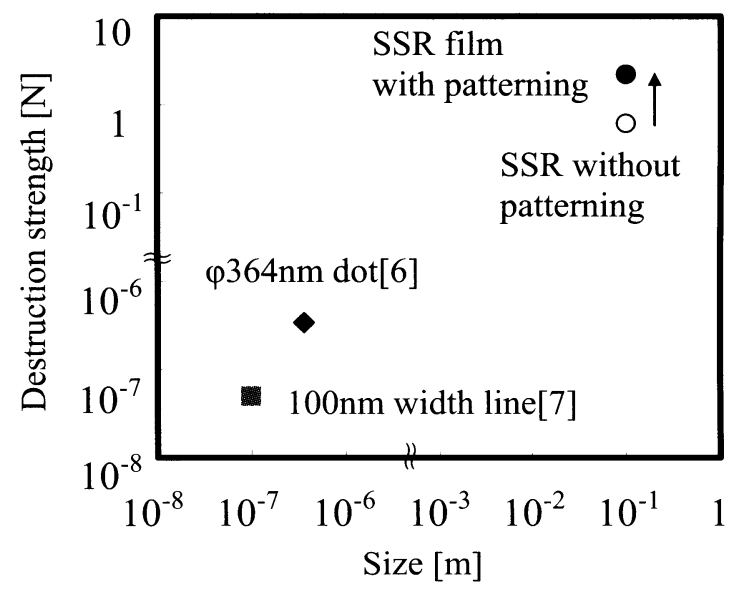

Fig.7 Destruction strength of structural materials.

Table2 Young's modulus values of various polymer materials.

\begin{tabular}{|c|c|}
\hline Polymer & $\begin{array}{c}\text { Young's Modulus, } \\
\text { E (GPa) }\end{array}$ \\
\hline Rubber & 0.001 \\
\hline Polyethylene & 0.15 \\
\hline Polystyrene & 2.1 \\
\hline HIPS & 2.5 \\
\hline PMMA & 2.5 \\
\hline Epoxy resin & 2.8 \\
\hline Rubber-toughened epoxy & 2.4 \\
\hline
\end{tabular}

of SSR film. It is considered that the tensile stress in the SSR film released at the hexagonal lattice structure. The SSR film with hard and flexible can be synthesized without hardening processes.

\section{Conclusion}

The SSR film with hexagonal lattice pattern is fabricated and characterized. The peel strength of SSR film with patterning is higher than that without patterning. It is confirmed that the SSR film with hexagonal lattice pattern is useful to apply to MEMS as a permanent material component.

\section{Acknowledgement}

The present work was partially supported by Grant-In-Aid Scientific Research from Japan Society for the Promotion of Science (Scientific Research (B) 19360157). 


\section{References}

1. K. Misumi, J. Photopolymer Sci. Technol., 19 (2006) 57.

2. R. Yang, Sensors and Actuators, A135 (2007) 625.

3. M. Han, Sensors and Actuators, A111 (2004) 14

4. Z. Ling, Microsyst. Technol., 13 (2007) 245

5. NIPPON KAYAKU Co., Ltd., Sample catalog, $\mathrm{p} 21$.

6. A. Kawai, J. Photopolymer Sci. Technol., 18 (2005) 729.

7. A. Kawai, J. Photopolymer Sci. Technol., 20 (2007) 825.

8. L. H. Sperling, "Introduction to Physical Polymer Science" 3rd edition, A John Wiley \& Sons, Inc. (2001) p609. 